\title{
Computational Biotechnology: An Approach in Silico Based Modeling Bioprocess
}

\author{
Reyed M Reyed* \\ Bioprocess Development Department, Genetic Engineering Biotechnology Research Institute "GEBRI”, City \\ For Scientific Research and Technology Application, SRTA-city, Alexandria, Egypt.
}

*Corresponding Author: Reyed M Reyed, Bioprocess Development Department, Genetic Engineering Biotechnology Research Institute "GEBRI”, City For Scientific Research and Technology Application, SRTA-city, Alexandria, Egypt.

\begin{abstract}
On-line estimation, monitoring and control of high non-linear and time variation bioprocesses is substantial significance to biotechnology as a means of realizing recovered output and lessen procedure unpredictability. Computational Statistics methodology including "monothetic analysis, fractional factorial designs , Response Surface Methodology and Computational Intelligence Techniques " Insilco technique”, including Artificial Neural Networks (ANNs), Genetic Algorithm (GA) and Differential Evolution (DE), can contribute significantly to the achievement of these objectives, Both strategies are suitable for Biotechnological Bioprocess Modeling, but differ in their extrapolation and interpolation capabilities on complex non linear fermentation biotechnology, and thus potentially conflict in their predictive accuracy. Each optimization technique has its own advantages and disadvantages. However, ANN has numerous benefits over conventional experimental or optimization methods. This review summarizes some of the recent computational modeling, can contribute significantly to the achievement of these objectives in the field of microbiome biotechnology, environmental bio-product for bioremediation by using bioprocess engineering. Biological engineering offers an smart substitute to conventional chemical production by using bioreactors as a factory for chemical synthesis (bio-preparation) a green and sustainable approach owing to its environmentally favorable properties and produce chemicals in a sustainable and stable manner.
\end{abstract}

Keywords: IN SILICO Microbiome, Bioprocess, Computational Statistics Methodology; Computational Intelligence Techniques.

White biotechnology, also referred to as industrial biotechnology, is biotechnology used in industrial processes. An example is an organism's model for the development of a useful chemical. Some other case in point is the use of certain enzymes as advanced manufacturing catalysts to either start producing valuable chemicals or bring down hazardous /polluting chemicals. White biotechnology needs lesser capital than conventional processes of manufacturing raw materials [1]. Fermentation biotechnology is a microbiome process through the function of enzymes that generates physiological changes in organic compounds. In microbiology, in the absence of oxygen, it is specifically defined as the mining of energy from carbs. It may extend more specifically to any system in which the interaction of microbiome contributes in a positive modification to a particular ingredient in the framework of food production [2]. Zymology is recognized as the process of fermentation. Fermentation of microbiome is the predominant means of generating ATP via anaerobically consuming natural resources [3]. Since the Iron age, humans have been using the fermentation to manufacture foodstuffs. For example, fermentation is being used for the conservation of lactic acid detected in sour food products such as pickled cucumbers, kimchi, and yogurt, as well as for the manufacturing of alcoholic beverages along with other liquor. Fermentation often happens throughout the gastrointestinal tracts of all animals, including humans.[4]. Microbiome fermentation is the justification for the enhanced development of a spectrum of vital chemicals, varying from sustenance, nanomaterials, pharmaceutical industries, larger quantities of chemical products, etc. In terms of economics, growth rates are the upper limit whenever the appropriate cultivation specifications, like those for nutritional demand, oxygenation, agitation, $\mathrm{pH}$, have been used. Within them, nutrition has 
been one of the crucial ecological factors that contribute a role in the development in the occurrence and aggregation of cannabinoid compounds, as its limitation not only inhibits microbiome growth but also has unique molecular and regulating implications. [5.6]. Furthermore, in terms of developing competent and value-effective fermentation technologies and therefore to achieve high material yields, it is also very important to establish satisfactory carbon-nitrogen and nutritional resources with other adequate components in the formulation of relevant manufacturing content. According to resources, $\mathrm{pH}$ and temperature inputs directly influence the microbiome's viability and growing levels throughout fermentation. Modifying the nutritional and the physicochemical parameters of fermentation is, therefore, necessary in order to enhance the microbiome biomass and incremental intermediate active ingredient biosynthesis [6]. For over two hundred years economic growth has contributed to effective and useful goods and services is a backbone of technological innovations. Due to increased knowledge and understanding, modern technological innovations and growing market expectations throughout this time, the underlying technology for digital biomanufacturing and industrialization has progressed. In past few years, an important division of biotechnology has been dedicated to building appropriate fermentation frameworks and proactive initiatives with the use of newly established fermentation innovations which is recently known by Bioprocess Engineering Utilizes micro-organisms to manufacture health and commercial biological ingredients- It deals with the design and testing of facilities and pathways for the research and development of product categories such as agricultural production, food, feed, pharmaceutical products, health supplements, chemical compounds, and nanomaterials. In bioprocess engineering, modeling reduces fermentation costs although it facilitates the analysis by means of various modeling parameters and provides process understanding that supports the operational strategy and subsequent automation and regulatory procedure. We have great potential to develop a high-performance, cost-effective and reliable model of computing that offers creative solutions to problems. If profitable, this biotechnology would make it easier to start producing green chemicals effectively on a large scale and preferably at low cost, with high titer, rate, and yield such as the biotechnological bioprocess strategy. Bioprocessing is also considered a green approach due to its eco-friendly properties as well as being sustainable. For example, in comparison to fossil combustion, the renewable feedstock can be used as an initial alternative energy source. In addition, bio-based chemical products can also acquire' cleaner' advantages compared to fossil-based replacements, which help reduce emissions of greenhouse gasses and the related impacts on global warming [7]. Given its environmental benefits, though, the fossilbased chemical industry cannot actually compensate for bioprocessing because of the cost and time required for the production of cell factories .and boost the production of antimicrobial products [8]. A critical step in the development of environmental microbiota for the manufacture of organic compounds of industrial significance. In reality, this approach is also used sometimes these days, Scientists basically, at the beginning of culture optimization media used single-factor -at -time experimentation. This classic method on the way to media optimization includes many repeated experiments to determine the ultimate conditions for microbiome growth and the highest biosynthesis of the organic compounds. The functional, or effective media constituents are typically costly, laborintensive, ineffective and time-wasting. Therefore, diverse microbiome pathways and variable responses to multiple different nutrients contribute to the current approach's inaccuracy. Consequently, with the prompt development of computer technology, several mathematical models for biotechnological bioprocess have been technologically advanced. Using various modeling algorithms and optimization of fermentation processes will contribute to an increased understanding of the process inputs for optimum yield and production rate. The main goal of modeling is to improve the procedures involved in manufacturing and improving the harvest of production a secondary metabolite in large number, furnishing immeasurable pharmacological and biological activities with higher value microbiomemetabolites such as like antibiotics, enzymes and probiotics, biopesticides, aromas, hormone, and an organic compound. To fulfill the need for living things need for new general formulations. the performance of bioprocess progress fermentation processes depends on many factors in terms of physicochemical environment $(\mathrm{pH}$, temperature, pressure) and process dynamics (feed rates). However, other key biological parameters such as biomass concentration, morphology, metabolic state, population heterogeneity, product concentration and quality, media composition. To achieve the best performance of fermentation processes. Bioprocesses engineering is described as 
biological systems that are non-linear, complex and unsteady; thus, it is challenging to develop a precise physical-based formula to characterize their physical performance. In addition, the development of accurate bioprocess models continues to baffle experts, as a result of the non-linear nature of the biochemical network interactions that occur during fermentation processes [9]. These engineering processes are influenced by several parameters which include $\mathrm{pH}$, temperature, hydraulic retention time, substrate concentration, etc.; therefore, the determination of the optimum values of these parameters is crucial [10]. Mathematical and statistical-based models can provide vital information for the understanding, analysis, and prediction of biological processes, and they are required for the optimization of key parameters in order to improve the process output [11] . Such bioprocess frameworks can also provide perspective into the individual as well as the dynamic impact on the destination output of specific input parameters. Furthermore, the use of certain bio-process models was constrained by non-linearity associated with microbiota fermentation. Like linear models, non-linear systems are not standardized resulting in contradictions between the observed tests. Implementation of bioprocess models that can easily accurately describe such non-linearity is of vital importance for bioprocess automation and scale-up. [12]. The computational model with the most cost-effective or highest achievable performance of fermentation process analyzes can lead to a better understanding of system inputs in order to ensure optimal production rate and yield. The most common approach model-based bioprocess design and optimization that is used by researchers in industrial experiments is (a) the classical one-variable-a-method, termed monothetical analysis is a system to design experiments that require testing factors or triggers, However, it never guarantees appropriate conditions for each particular variable. Such drawbacks can be addressed by computational techniques utilizing single variable modeling. Two hypotheses are practicable with empirical approaches, i.e. Statistical and artificial black-box approach based on intelligence. (b) Binomial variable patterns are conceptual models comprising a subset (fraction) of the investigational loops of a total factorial design. The subset is chosen so as to exploit the sparsity (also well-known by data sparseness, data paucity, etc) of effects principle to expose Data about one of the most important characteristics of the issue evaluated in terms of processing loops and resources that used a fraction of the effort of a comprehensive factorial design. in certain phrases, it takes advantage of the fact that almost all fully factorial simulations are sometimes redundant with very little new system data (c) Response Surface Methodology "RSM" [13]with is association a Central Composite Design (CCD)[14] and A Box-Behnken design explores the relationships between several explanatory variables and one or more response variables. A Response Surface Design is a set of advanced fractional factorial techniques that help you better understand and optimize your response. Response Surface Design methodology is often used to improve models after you have determined important factors using screening designs or factorial designs; especially if you suspect curvature in the response surface. The difference between a response surface equation and the equation for a factorial design is the addition of the squared (or quadratic) terms that lets you model curvature in the response, making them useful for A) Understanding or mapping a region of a response surface. Response surface equations model how changes in variables affect the response of interest. B) Finding the levels of variables that optimize a response. Selecting the operating conditions to meet specifications. Another aim of RSM is to identify and optimize significant factors, with the purpose of determining what levels of these factors maximize, the response (product yield productivity). It uses empirical model frameworks are also used to design mathematical designs that link response (contingent parameter) with other variables (independent parameters) [15]. Recently, Bioprocesses, Bioengineering sectors are moving towards digitalization and automation. In this age of a new generation of bioprocessing intelligent industrial technology, Machine-to-Machine communication, this numerical interaction between and within machines and systems is the cyber-physical heart of the fourth industrial revolution (IV). It is indeed a combination of innovation of machine learning, computer vision, Internet of things, 3D digitizing, bioengineering, artificial intelligence, and other computerization network technology, The IV Green and sustainable revolution is much more than technological progress. The genuine opportunity is to have the state of the art over and above. The conventional era of bioprocess scenario is now disappearing and gradually being replaced by Artificial intelligence tools have emerged as a promising method for modeling and optimization of bioprocess engineering Some of these include artificial neural network (ANN), which are considered suitable in the design of 
Insilco bioprocesses for research and development. Complex dynamic systems containing living cells or active enzymes out of intact cells are becoming manageable through automated on-line analysis and types of software for feed control, predefined process optimizations strategies and highly versatile sample processing. Empirical strategies in biotechnology and bioprocess development are replaced by systematic protocols for experimentation. Today extra- and intracellular enzyme activity can be measured on-line. Metabolic flux dynamics of metabolites are quantitatively calculated in a noninvasive strategy and perfection of analytical precision accuracy and reproducibility has turned the earlier descriptive biology into a hard science-quantifiable like physics and chemistry. Recently, Quantitation is gradually replacing the qualitative models - a development which is necessary for deeper insight into living systems. Quantitation provides the tools to overcome the limits of a pure description of the molecular basis of life. It is clear that the informatics part in life sciences is growing in importance. Before and a chemostat produced some 40-50 signals of physical, chemical or biological process parameters or state variables. At least 1 megabyte per day had to be processed. Recently data flux has increased several-fold. It is still expanding and enormous computer capacities and software demand are expected for the future and progressively increasing number of researchers have started to build up high-tech equipment, with the enhanced analysis used in off- and on-line mode. Other groups have begun to study biological problems at a molecular level, like regulation of metabolic or cell cycle mechanisms, cell structures, the behavior of recombinant strains or animal cells in submerged cultures. They are involved in bioprocess optimization and advanced control, production improvement strategies and modeling, simulation and image analysis. [16]. So far. In the last two decades, ANN has been developed and extensively used by many researchers in fermentation optimization. ANN are a computer-based problem-solving systems of evolutionary computation field based on the principle of evolution theory and has emerged as an attractive tool for non-linear multivariate modeling. [17]. the advantage of ANN is the standard framework and has the ability to solve problems from background information. The main advantages and better quality of the tested ANN are: (I) ANN does not need an adequate practical pre-specification and (ii) ANN has a generic modulation ability, i.e. almost all non-linear functions and algebraic functions can be computed, whereas RSM is successful only for logarithmic approaching variables. RSM is still the most common method for improving fermentation media. It is one of the efficient methods for non-linear optimization. But its main limitation of RSM is that is assuming an only quadratic non-linear correlation. So if we want to use RSM effectively, we need to narrow down the search window appropriately (if we get smaller the look for window contracted enough, the linear correlation may also be sufficient). This makes the search process highly dependent on search space. It will require either extra experiments or good priory knowledge of the system to fix the search window. Since ANN can inherently capture almost any form of non-linearity, it can easily overcome the abovediscussed limitation of RSM. Thus, in the case of ANN, a more liberal search space can be chosen; even if, the correlations in that search space are more complex than quadratic. Some studies have comparatively examined the use of ANN and RSM for the design of mathematical models and improving biotechnological production bioprocesses [18], [19], [20]. The fast improvement of computational tools is the major motivation following the wide appliance of ANNs in investigates and progress. At this time, ANNs are engaged in the anticipation of a variety of results including all fields of biotechnology. The application of ANNs as a modeling tool stems from their astonishing information-processing features which are attributed primarily to non-linearity, high parallelism, fault, and noise acceptance as well as their learning and generalization abilities. In contrast to traditional modeling tools, ANNs offer a model-free, adaptive, parallel- processing and vigorous elucidation with error and failure tolerance. Furthermore, their knowledge competence for treating erroneous and unclear statistics and their capability to oversimplify invisible designs are faultless. As emphasized in this mini-review, ANNs have exposed a superior expectation precision-matched up to other modeling approaches such as RSM. This tendency has been reported above. The achievement of these models for future studies on bioprocesses application will help reduce costs and duration during process development."The Fourth digital transformation and Industrialization revolution is stagnant and still in its growing and emerging state. But with the fast rate of progression and interference to the tech world and community, the period to link in is nowadays." 


\section{REFERENCES}

[1] Frazzetto G ( 2003). "White biotechnology ".EMBO Reports. 4 (9): 835 7.doi:10.1038/sj.embor.embor928. PMC 1326365.PMID 12949582.

[2] Hui, Y. H. (2004).Handbook of vegetable preservation and processing. New York: M. Dekker. p. 180.ISBN 978-0-8247-4301-7.OCLC 52942889.

[3] Klein, Donald W.; Lansing M.; Harley, John (2006). Microbiology (6th ed.). New York: McGraw-Hill. ISBN 978-0-07-255678-0.

[4] Bowen, Richard."MicrobiomeFermentation". Hypertexts for biological sciences. Colorado State University. Retrieved 29 April 2018.

[5] Doull JL, Vining LC. (1990). Nutritional control of actinorhod in production by Streptomyces coelicolorA3(2): suppressive effects of nitrogen and phosphate. Appl Microbiol Biotechnol 32:449-54

[6] Selvanathan Lathaa, Govindhan Sivaranjania and Dharuma durai Dhanasekaran Critical Reviews in Microbiology, (2017)VOL. 43, NO. 5, 567-582.

[7] Moore, R.H. et al. (2017) Biofuel blending reduces particle emissions from aircraft engines at cruise conditions. Nature 543, 411-415.

[8] Galanie, S. et al. (2015) Complete biosynthesis of opioids in yeast. Science 349, 1095-1100.

[9] Franco-Lara E, Link H, Weuster-Botz D. Evaluation of artificial neural networks for modeling and optimization of medium composition with a genetic algorithm. Process Biochem. 2006;41:2200-2206.

[10] Wang JL, Wan W. Factors influencing fermentative hydrogen production: a review. Int J Hydrog Energy. 2009;34:799-811.

[11] Escamilla-Alvarado C, Rios-Leal E, Ponce-Noyola MT, et al. Gas biofuels from solid substrate hydrogenic- methanogenic fermentation of the organic fraction of solid municipal wastes. Process Biochem. 2012;47 (11):1572-1587.

[12] Ahmadian-Moghadam H, Elegado FB, Nayve R. Prediction of ethanol concentration in biofuel production using artificial neural networks. Am J Model Optim. 2013;1(3):31-35.

[13] Soad abdel galil, Ahmed M Attia, Reyed Reyed, Nadia A Soliman, Hesham A El Enshasy, Application of Experimental Designs for Optimization the Production of Alcaligenes Faecalis Nyso Laccase" Journal of Scientific \& Industrial Research Vol. 77, December 2018.

[14] Soad abdel galil, Ahmed M Attia, Reyed Reyed, Nadia A Soliman. "Application of Central Composite Designs Utilized for Scale-up Strategies in the Optimization Process for Development Laccase Production and for the Bioremediation of Various Industrial Pollutants". Acta Scientific Microbiology 2.11 (2019): $18-27$.

[15] Badia Dandach Bouaoudat, Farouk Yalaoui, Lionel Amodeo \& Françoise Entzmann (2012) Efficient Developments in Modeling and Optimization of Solid State Fermentation, Biotechnology \& Biotechnological Equipment, 26:6, 3443-3450

[16] Preface Journal of Biotechnology 52 (1997) 171-172.

[17] Kiran M. Desai, Shrikant A. Survase, Parag S. Saudagar, S.S. Lele, Rekha S. Singhal Comparison of artificial neural network (ANN) and response surface methodology (RSM) in fermentation media optimization: Case study of fermentative production of scleroglucan / Biochemical Engineering Journal 41 (2008) 266-273.

[18] Wang JL, Wan W. Optimization of fermentative hydrogen production process using genetic algorithm based on neural network and response surface methodology. Int J Hydrog Energy. 2009;34:255-261.

[19] Whiteman JK, Gueguim Kana EB. Comparative assessment of the Artificial Neural Network and Response Surface Modelling efficiencies for biohydrogen production on sugar cane molasses. Bioenergy Res. 2013;7(1):295- 305.

[20] Esfahanian M, Nikzad M, Najafpour G, et al. Modeling and optimization of ethanol fermentation using Saccharomyces cerevisiae: response surface methodology and artificial neural network. Chem Ind Chem Eng Q. 2013;19(2):241-252.

Citation: Reyed M Reyed, " Computational Biotechnology: An Approach in Silico Based Modeling Bioprocess.", International Journal of Research Studies in Microbiology and Biotechnology (IJRSMB), vol. 5, no. 4, pp. 1-5, 2019. Available: DOI: http://dx.doi.org/10.20431/2454-9428.0504001

Copyright: (1) 2019 Authors. This is an open-access article distributed under the terms of the Creative Commons Attribution License, which permits unrestricted use, distribution, and reproduction in any medium, provided the original author and source are credited. 